\title{
Editor's Note: Special Issue on Big Data and Smart Computing
}

The World Wide Web: Internet and Web Information Systems journal gratefully acknowledges the editorial work of the following scholars on this special issue "Big Data and Smart Computing":

Wookey Lee, INHA University.

Wook-Shin, Han Pohang University of Science and Technology.

The 6 papers in this issue include:

"Ecosystem on the Web: non-linear mining and forecasting of co-evolving online activities" by Yasuko Matsubara, Yasushi Sakurai, and Christos Faloutsos.

"An efficient key management scheme for user access control in outsourced databases" by Seungtae Hong, Hyeong-Il Kim, and Jae-Woo Chang.

"MTP: discovering high quality partitions in real world graphs" by Yongsub Lim, Won-Jo Lee, Ho-Jin Choi, U Kang.

"Para-G: Path pattern query processing on large graphs" by Yiyuan Bai, Chaokun Wang, and Xiang Ying.

"Set-based unified approach for summarization of a multi-attributed graph" by Kifayat Ullah Khan, Waqas Nawaz, and Young-Koo Lee.

"Finding efficiencies in frequent pattern mining from big uncertain data" by Carson Kai-Sang Leung, Richard Kyle MacKinnon, and Fan Jiang. 